\title{
Developing Preliminary Scales for Assessing the HiTOP Detachment Spectrum
}

\author{
Johannes Zimmermann' (iD, Thomas A. Widiger², Lara Oeltjen³, \\ Christopher C. Conway ${ }^{4}$, and Leslie C. Morey ${ }^{5}$
}

\begin{abstract}
The Hierarchical Taxonomy of Psychopathology (HiTOP) is an empirical-based classification of psychopathology. Detachment is one of the six spectra in the current HiTOP working model. The aim of this study was to develop preliminary scales for the HiTOP Detachment spectrum that can be used in the next phase of developing a comprehensive measure of HiTOP. We had 456 participants from MTurk (Sample I) and 266 university students (Sample 2) complete an online survey including a pool of 247 Detachment items assessing 15 consensually defined low-order constructs. Using a stepwise procedure involving factor analyses and ant colony optimization methods, we developed seven 8-item scales that capture unipolar facets of Detachment: anhedonia, suspiciousness, social withdrawal, intimacy avoidance, unassertiveness, risk aversion, and restricted affectivity. Three other 8-item scales emerged that tapped into a Maladaptive Extraversion construct (attention-seeking, thrill-seeking, and domineering), which was mostly unrelated to unipolar Detachment in factor analyses. The 10 scales were unidimensional, reliable, and showed some evidence of convergent and discriminant validity. We discuss challenges of assessing Detachment when moving forward with developing a comprehensive measure of HiTOP.
\end{abstract}

\section{Keywords}

Hierarchical Taxonomy of Psychopathology, personality, detachment, extraversion, factor analysis, ant colony optimization, scale construction

The authoritative diagnostic nomenclature of psychopathology is provided by the American Psychiatric Association's (2013) Diagnostic and Statistical Manual of Mental Disorders (DSM), consisting largely of categorical syndromes identified within clinical practice. Recently, the Hierarchical Taxonomy of Psychopathology (HiTOP) initiative has proposed an alternative classification system comprising hierarchically organized dimensions that are derived from empirical correlations of traits, signs, and symptoms (Kotov et al., 2017). At one level of the current HiTOP working model are six spectra: Somatoform, Internalizing, Thought Disorder, Externalizing-antagonistic, Externalizing-disinhibited, and Detachment. One important next step of the HiTOP initiative is to develop a comprehensive measure that captures individual differences in all six of these domains. To achieve this goal, the HiTOP Measures Development Workgroup (MDW) set up a process in which subworkgroups first develop scales separately for each spectrum (Phase 1) and then all newly constructed items are jointly applied in large samples (Phase 2; Simms et al., in press). Here, we present the results of Phase 1 of the HiTOP Measures Development Sub-Workgroup (MDSW) for Detachment. That is, our goal was to develop preliminary scales for Detachment that can be used in the next phase of data collection.

According to the first draft of the HiTOP working model, Detachment is manifested in anhedonia, depressivity, intimacy avoidance, suspiciousness, withdrawal, interpersonal passivity, disaffiliativeness and (low) attention seeking (Kotov et al., 2017). Detachment is aligned with introversion (vs. extraversion), one of the five fundamental domains of general personality included within the five-factor model (Costa \& McCrae, 1992). Detachment is also included in most models of maladaptive personality traits. Specifically, it is present within the DSM-5 Section III trait model (with facets of withdrawal, intimacy avoidance, anhedonia, depressivity, restricted affectivity, and suspiciousness;

\footnotetext{
'University of Kassel, Kassel, Germany

${ }^{2}$ University of Kentucky, Lexington, KY, USA

${ }^{3}$ University of Jena, Jena, Germany

${ }^{4}$ Fordham University, Bronx, NY, USA

${ }^{5}$ Texas A\&M University, College Station, TX, USA

Corresponding Author:

Johannes Zimmermann, Department of Psychology, University of Kassel, Holländische Str. 36-38, Kassel 34127, Germany.

Email: jz@uni-kassel.de
} 
American Psychiatric Association, 2013, p. 779) as well as within the trait model of the recent International Classification of Diseases (ICD-11) (defined as the tendency to maintain interpersonal and emotional distance; World Health Organization, 2018). Detachment was also included within the trait models that preceded DSM-5, such as the Dimensional Assessment of Personality Pathology (DAPP; Livesley et al., 1998), identified therein as Social Avoidance, with the facets of intimacy problems, low affiliation, and restricted expression, and within the Schedule of Nonadaptive and Adaptive Personality (SNAP; Clark, 1993) identified as low Positive Temperament, with the single facet of detachment (alongside other facets that defined high positive temperament). Detachment is also evident in other contemporary personality models, such as (a) the Computerized Adaptive Test of Personality Disorder (CAT-PD; Wright \& Simms, 2014), identified as low Positive Emotionality with the facets of anhedonia, social withdrawal, emotional detachment, and romantic disinterest; and (b) the five-factor model of personality disorder (FFMPD; Widiger et al., 2012), identified as introversion, with the facets of social isolation and withdrawal, social anhedonia, physical anhedonia, detached coldness, risk aversion, unassertiveness, joylessness, shrinking, and social dread. It is perhaps noteworthy that, besides the HiTOP working model, only the DSM-5 trait model includes suspiciousness and depressivity within Detachment (Watson et al., 2013; Widiger, 2011).

\section{Selection of Constructs for the HiTOP Detachment Domain}

Each of these trait models is, of course, assessed by a corresponding self- or informant-report inventory, but consistent with the general principles of the HiTOP MDW (Simms et al., in press), it was decided not to give undue authority to any particular existing instrument for the assessment of HiTOP. In addition, the eventual validation of HiTOP scales requires the consideration of their convergent, discriminant, and structural relationship with existing measures and models. Therefore, each of the four Detachment MDSW members nominated constructs to include within the HiTOP detachment spectrum. Erring in the direction of overinclusiveness rather than underinclusiveness, all constructs nominated by at least two subworkgroup members were included. Eleven constructs emerged: anhedonia, restricted affectivity, autism, risk aversion, coldness, unassertiveness, intimacy avoidance, apathy, alienation, social withdrawal, and suspiciousness. Note that social anxiety was not nominated by at least two work group members - only one member nominated shyness. The decision not to include social anxiety seems understandable, as social anxiety is clearly associated with anxiousness and seems to be better located in the Internalizing spectrum (Watson et al., in press).
All of the HiTOP spectra are defined in a unipolar fashion, not recognizing maladaptive traits that are on the opposite pole of a respective domain. This is consistent with the DAPP and for the most part with the DSM-5 Section III (with the exception of rigid perfectionism that is conceptualized as opposite to the domain of Disinhibition; American Psychiatric Association, 2013). However, other trait models have recognized maladaptive traits opposite to the domain of Detachment. The SNAP includes entitlement and exhibitionism; the CAT-PD includes exhibitionism and grandiosity; and the FFMPD includes exhibitionism, thrill-seeking, dominance, intimacy-seeking, and attention-seeking. Indeed, even though no such instruction was provided to the four MDSW members, four maladaptive traits opposite to Detachment were nominated by at least two members: exhibitionism, attention-seeking, domineering, and thrill-seeking. However, it was quite possible that these opposite pole trait domains would not perform structurally as expected (i.e., loading in the opposite direction on a Detachment factor) given that maladaptive traits opposite to one another may at times even correlate positively with one another, in part due to the impact of shared aspects of maladaptivity (Crego et al., 2020). That is, these shared aspects may offset an otherwise inverse relationship between the respective traits (Widiger \& Crego, 2019).

MDSW members reached a consensus on the definition of each of the 15 constructs (see Table 1). Draft items were then submitted by each subworkgroup member, resulting in up to 20 items for each scale. The items were written in the present tense and included a mix of statements reflecting personality traits relevant to the spectrum (e.g., "I prefer to be by myself") and specific symptoms or behaviors (e.g., "I have no interest in my usual social activities."). These lists were reduced by the subworkgroup chair (Dr. Widiger) on the basis of obvious redundancy, as well as an effort to reduce the number of items that would be reverse-keyed. The inclusion of reverse-keyed items has been a longstanding tradition in personality assessment, in large part to address a potential response style of acquiescence. However, there is a growing literature to suggest that reverse-worded (or reverse-keyed) items may perform consistently worse relative to positively worded items (Crego \& Widiger, 2014; Woods, 2006). The reasons are not yet entirely clear, but one common explanation is that not endorsing the presence of a trait (e.g., responding negatively to the item, "I am socially distant from others") does not necessarily indicate the presence of the opposite trait ("I am closely involved with others"). Finally, because certain Detachment constructs may be also relevant for the Thought Disorder spectrum, the chairs of these two subworkgroups compared conceptual definitions and determined five overlapping constructs, including (low) exhibitionism, anhedonia, social withdrawal, intimacy avoidance, and suspiciousness (Cicero et al., in press). 


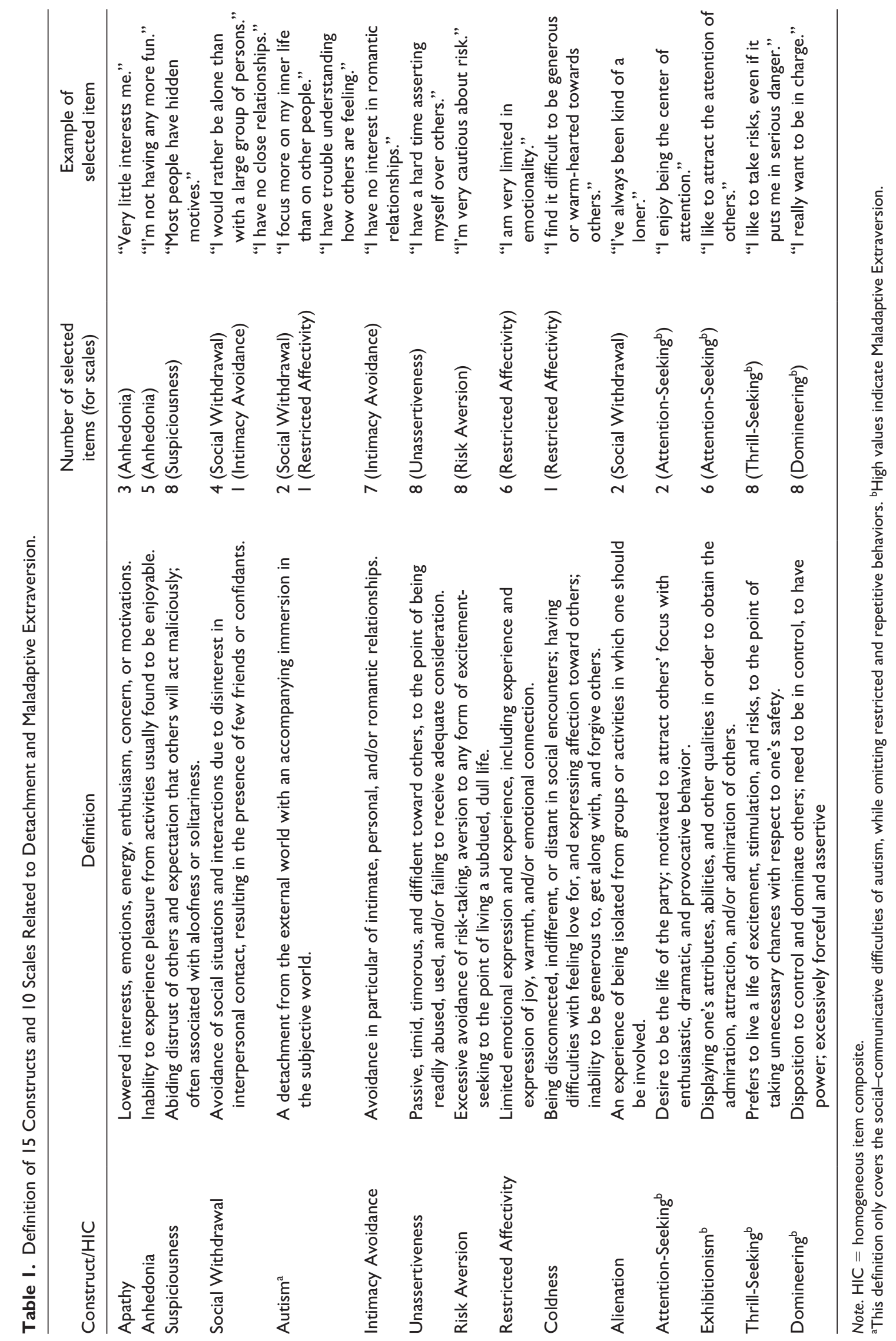


All unique items for these five constructs, whether originally written for the Detachment or Thought Disorder spectrum, were included. The final list comprised 247 items, including 12 reverse-keyed items.

\section{The Current Study}

The aim of this study was to evaluate and refine these 15 constructs and develop preliminary scales for the HiTOP Detachment domain. To this end, we had 456 participants from MTurk (Sample 1) and 266 university students (Sample 2) complete an online survey including the 247 Detachment items. First, we identified the preliminary domain structure by factor-analyzing the total scores of the 15 constructs that served as indicators for "homogeneous item composites" (HICs). Second, we identified preliminary scales within each HIC-based factor using item-level exploratory factor analysis (EFA). Third, we selected an optimal set of eight items for each preliminary scale using an ant colony optimization (ACO) algorithm (Leite et al., 2008; Schroeders et al., 2016) and provided first information on the scales' reliability and validity. We report how we determined our sample size, all data exclusions, all manipulations, and all measures in the study.

\section{Method}

\section{Procedure}

Our goal was to recruit two samples of at least 250 participants, as this is the minimum sample size to obtain stable estimates of correlations (Schönbrodt \& Perugini, 2013). Participants of Sample 1 were recruited via the crowdsourcing platform Mechanical Turk (MTurk). Our survey was published on the MTurk marketplace on May 26th, 2019 with the following keywords tailored to the nonprofessional target group: psychology, personality, individual differences, thoughts, feelings, behavior, and research. Workers were invited to participate if they were (a) at least 18 years old, (b) located in the United States, and (c) had an MTurk approval rate of more than $95 \%$. Five hundred and twenty-six workers completed the survey and received the compensation of $\$ 4$. Data for Sample 2 were collected from students of the College of William and Mary, in Williamsburg, Virginia, during the Spring of 2019. Three hundred and twenty-one students completed the survey. All students were compensated with academic course credit.

The questionnaire for both samples consisted of sections on sociodemographic information (age, gender, education, nationality, native language, and history of treatment for mental disorders), detachment, normal-range personality traits, and maladaptive personality trait facets. We did not collect data on ethnic or racial identity. Within each survey section, items were presented in a randomized, unique order for each participant.
To identify careless responding, we included an additional self-report scale on study engagement proposed by Meade and Craig (2012). The scale consists of 10 items on diligence and interest (e.g., "I carefully read every survey item"), which are rated on a 5-point scale from 1 (strongly disagree) to 5 (strongly agree). As careless responding might not always be admitted, we followed the recommendations of Meade and Craig (2012) and additionally included three bogus items in the section on detachment. Of the 10 bogus items suggested by Meade and Craig, we selected the three items that best matched the response format of the detachment items (e.g., "I have been to every country in the world"). We excluded participants of both samples with more than $10 \%$ missingness (i.e., $\geq 35$ items), a response of 2 or greater to one of the three bogus items (on a scale from 0 to 3 ) or a mean value on the diligence scale below the theoretical midpoint $(<3)$.

\section{Samples}

Sample I. Application of the exclusion criteria resulted in a final development sample of 456 MTurk workers, hence, 70 participants $(13.3 \%)$ were excluded due to incomplete or careless responding. In our final sample, $229(50.2 \%)$ of the participants were female, $224(49.1 \%)$ male, and 2 nonbinary (1 missing) with a mean age of 41.5 years $(S D=12.5$, range = 21-74). Participants completed an average of 15.3 years of education $(S D=2.9$, range $=3-36)$. The vast majority $(96.1 \%)$ reported to be native English speakers. A total of 335 (73.5\%) of them stated that they never received any medical or psychotherapeutic treatment for mental disorders, $114(25 \%)$ declared they did, and 7 declined to answer this question.

Sample 2. We had to exclude 55 participants (17.1\%) from our student sample due to incomplete or careless responding, resulting in a final validation sample of 266 university students. Indeed, 192 (72.2\%) of them were female, 73 (27.4\%) male, and 1 nonbinary with a mean age of 19.0 years $(S D=$ 1.2 , range $=18-27)$. However, $89.8 \%$ of the sample indicated English as their native language. Forty-three (16.2\%) students reported a history of mental health treatment.

\section{Measures}

HiTOP Detachment Phase I Item Set. As outlined above, constructs nominated by at least two members of the HiTOP MDSW were included within the preliminary formulation of the detachment spectrum. Of the 15 included constructs, 11 were "positively keyed" and 4 were opposite to detachment (see Table 1). The final list comprised 247 items with a minimum of 14 items (apathy, anhedonia, domineering) to a maximum of 23 items (suspiciousness) per construct. Twelve of the items were reverse-keyed with respect to their construct. In line with the guidelines of the HiTOP 
MDW (Simms et al., in press), we used the following instruction: "Please consider whether there have been significant times during the last 12 months during which the following statements applied to you." Participants rated each item on a 4-point degree-based response format of 0 (not at all), 1 (a little), 2 (moderately), and 3 (a lot).

Personality Inventory for DSM-5 Short Form (PID-5 SF). The PID-5 (Krueger et al., 2012) is a self-report inventory developed for the assessment of the 25 maladaptive personality trait facets of the DSM-5 Section III trait model. The 220 items (e.g., "I keep my distance from people") are answered on a 4-point scale from 0 (very false or often false) to 3 (very true or often true). The presented study is based on the abbreviated 100-item version by Maples et al. (2015) that reduces the original set of items to four items per trait facet. Our survey only included the 24 items of the six detachment facets: suspiciousness, anhedonia, depressivity, withdrawal, restricted affectivity and intimacy avoidance.

Big Five Inventory-2 (BFI-2). The BFI-2 (Soto \& John, 2017) is an enhanced version of the Big Five Inventory (John et al., 1991), which represents each of Big Five Personality domains (Extraversion, Conscientiousness, Agreeableness, Negative Emotionality, and Open-Mindedness) by three trait facets. The inventory consists of 60 statements (e.g., "I am someone who ... is outgoing, sociable") that are rated on a 5-point scale from 1 (disagree strongly) to 5 (agree strongly). To control for acquiescence, it comprises an equal number of true- and false-keyed items.

\section{Statistical Analyses}

In line with the recommendations of the HiTOP MDW (Simms et al., in press), we analyzed the data in three steps to identify (a) the preliminary (higher-order) domain structure, (b) preliminary (low-order) scales, and (c) preliminary item sets for each scale. After this, we explored the reliability and validity of the newly developed scales. Our analyses were mostly based on data from Sample 1. We used Sample 2 only for testing the robustness of the new scales' psychometric properties. All analyses were conducted on the statistical platform R (R Core Team, 2016), using the packages psych (Revelle, 2018), mirt (Chalmers, 2012), lavaan (Rosseel, 2012), and stuart (Schultze, 2019).

Analytic Step I. We scored the 15 HICs by averaging item responses within each HIC, and then conducted an EFA with oblique rotation of the 15 scores using maximum likelihood estimation as implemented in the psych package. The goal was to identify smaller sets of related constructs within the spectrum that should be subjected to item-level analyses in the next step. We used parallel analysis to guide on the number of factors to be extracted. The standardized factor loading threshold for including a HIC on a factor was .50. If a HIC loaded on multiple factors, we chose the factor on which the HIC loaded most strongly.

Analytic Step 2. We conducted item level EFAs within each of the broad factors identified in Step 1. To account for the ordinal scaling of the items, we factor-analyzed the polychoric correlations matrices using the psych package. Again, parallel analyses were used to set the number of factors to be extracted. In line with the HiTOP MDW guidelines, the loading threshold for item inclusion on a factor was .40 (i.e., convergent validity), and the minimum difference between primary and secondary loadings was .20 (i.e., discriminant validity). The resulting items formed the preliminary scales that had to be shortened in the next step.

Analytic Step 3. We selected an optimal set of eight items for each scale, corresponding to the minimum number of items per scale specified by the HiTOP MDW guidelines. Item selection was conducted using an ACO algorithm. The ACO method is particularly suitable for developing short versions of longer scales and is arguably superior to conventional item selection strategies such as selecting items based on the corrected part-whole correlation (Leite et al., 2008; Olaru et al., 2019; Schroeders et al., 2016). It is based on an iterative process in which combinations of items are repeatedly selected and evaluated using a preestablished optimization criterion. Items from favorable combinations are marked, which increases the probability that these items will be selected in subsequent runs. As an optimization criterion, we combined several criteria for reliability and model fit (cf. Schroeders et al., 2016). The criteria were derived from a one-dimensional confirmatory factor analysis (CFA) model with all items loading on the latent factor and residual correlations fixed to zero. In particular, reliability was defined by (a) McDonald's Omega of the scale and (b) the minimum factor loading across items, and model fit was defined by (c) the root mean square error of approximation (RMSEA) and (d) the comparative fit index (CFI). Models were estimated using the WLSMV estimator based on the polychoric correlation matrix. The four individual criteria were transformed and then combined into a single optimization criterion. The exact formulas and further specifications of the ACO algorithm are presented in the online Supplemental Material. These analyses were conducted using the packages lavaan and stuart. For the final eightitem scales, we computed item response theory (IRT) parameters of graded response models using the package mirt. To examine convergent and discriminant validity, we computed correlations of scales' total scores with detachment-related facet scores of the PID-5 SF and the domain scores of the BFI-2. In addition, we analyzed the psychometric properties of the final scales in Sample 2. 


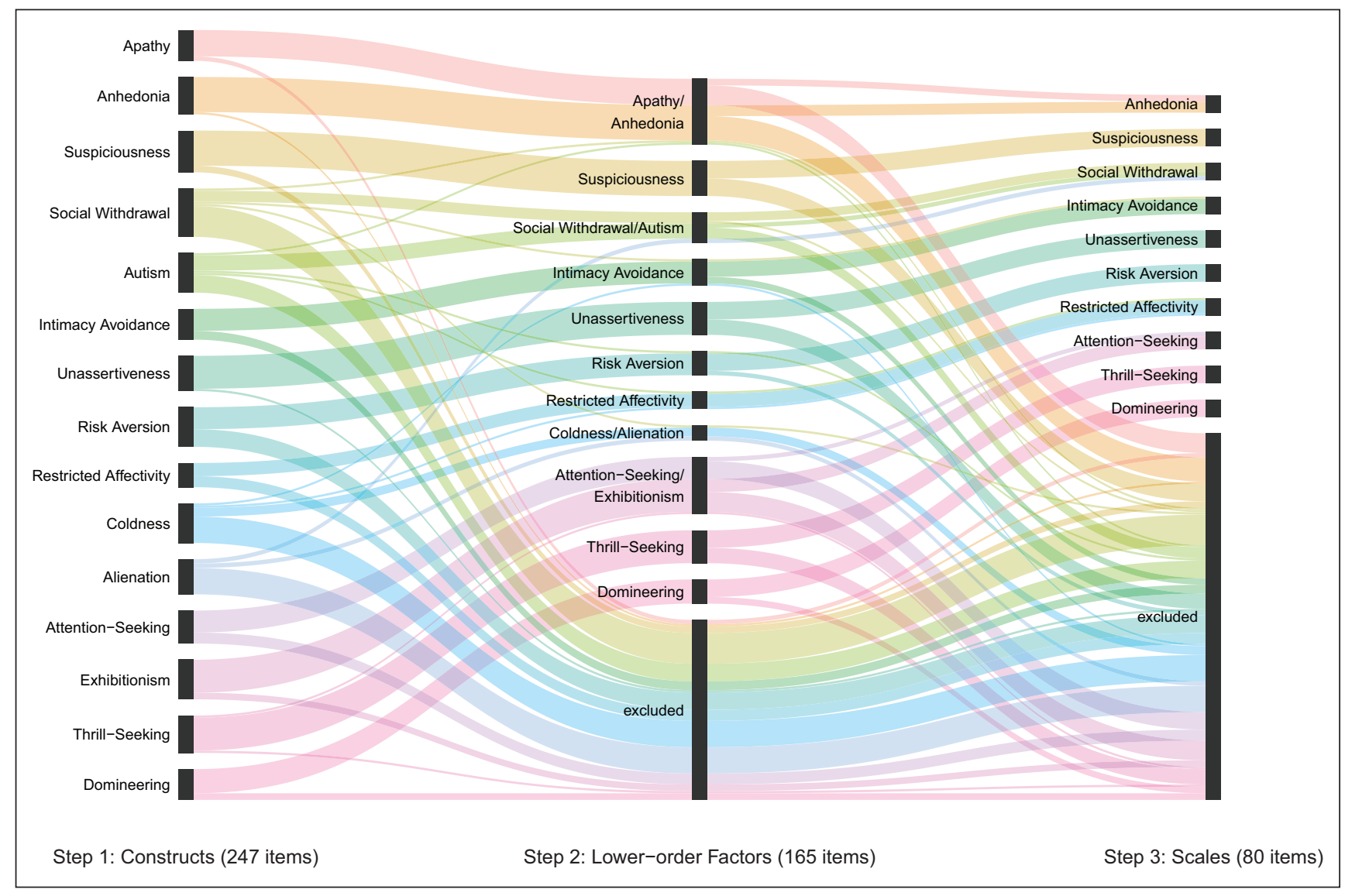

Figure I. Process of item selection from the constructs (Analytic Step I) via the low-order factors (Analytic Step 2) to the scales (Analytic Step 3).

\section{Results}

\section{Analytic Step I}

Parallel analysis suggested extracting two broad factors from the 15 HIC scores (see online Supplementary Figure 1). Considering a primary loading greater than .50 as substantial, the first factor (F1: Detachment) comprised risk aversion, anhedonia, alienation, social withdrawal, coldness, intimacy avoidance, unassertiveness, autism, suspiciousness, restricted affectivity and apathy, and the second factor (F2: Maladaptive Extraversion) comprised thrill-seeking, exhibitionism, domineering, and attention-seeking (see online Supplemental Table 1). That is, constructs defining opposite poles of the detachment domain loaded on two separate factors. The two factors were slightly negatively correlated, $r=-.08$.

\section{Analytic Step 2}

We combined the items of the HICs pertaining to the two broad factors established in Step 1, resulting in a pool of 183 items for F1, and 64 items for F2. Parallel analyses suggested extracting eight low-order factors for F1, and four low-order factors for F2 (see online Supplemental Figure 2). For F1, 113 items fulfilled the inclusion criteria of a convergent validity greater than .40 and a discriminant validity greater than .20 . The low-order factors (i.e., preliminary scales) were apathy/anhedonia (30 items), suspiciousness (16 items), social withdrawal/autism (14 items), intimacy avoidance (12 items), unassertiveness (15 items), risk aversion (11 items), restricted affectivity ( 8 items), and coldness/alienation ( 7 mostly reverse-keyed items; see Figure 1). The correlations between the low-order factors were all positive $(.08<r<.56)$. For F2, 52 items fulfilled the inclusion criteria. The low-order factors were attentionseeking/exhibitionism (26 items), thrill-seeking (15 items), and domineering (11 items; see Figure 1). No item related to the fourth factor fulfilled the inclusion criteria. The correlations between the low-order factors were again all positive $(.12<r<.59)$.

\section{Analytic Step 3}

We decided to omit the preliminary scale coldness/alienation, because this factor included mostly reverse-keyed 
Table 2. CFA Model Fit and Reliability of HiTOP Scales in Sample I.

\begin{tabular}{|c|c|c|c|c|c|c|c|c|}
\hline Domain & $\chi^{2}$ & $p$ & CFI & TLI & RMSEA & SRMR & $\alpha$ & $\omega^{\mathrm{cat}}$ \\
\hline Anhedonia & 16.195 & .704 & I & I & 0 & .009 & .950 & .953 \\
\hline Suspiciousness & 47.095 & .001 & .995 & .993 & .055 & .028 & .902 & 911 \\
\hline Social withdrawal & 46.208 & .001 & .997 & .996 & .054 & .022 & .923 & .934 \\
\hline Intimacy avoidance & 75.169 & $<.001$ & .993 & .99 & .078 & .033 & .908 & .928 \\
\hline Unassertiveness & 28.201 & .105 & .998 & .997 & .030 & .023 & .865 & .873 \\
\hline Risk aversion & 72.270 & $<.001$ & .994 & .992 & .076 & .022 & .927 & .934 \\
\hline Restricted affectivity & 163.910 & $<.001$ & .972 & .960 & .126 & .053 & .895 & .922 \\
\hline Attention-seeking & 12.069 & .914 & I & 1.002 & 0 & .012 & .910 & .917 \\
\hline Thrill-seeking & 32.891 & .035 & .998 & .997 & .038 & .022 & .901 & .906 \\
\hline Domineering & 22.011 & .340 & I & I & .015 & .014 & .920 & .929 \\
\hline
\end{tabular}

Note. $N=456$. All models have 32 parameters and 20 degrees of freedom. CFI $=$ comparative fit index. TLI $=$ Tucker-Lewis index. RMSEA $=$ root mean square error of approximation. SRMR $=$ standardized root mean square residual. $\alpha=$ Cronbach's alpha. $\omega^{\text {cat }}=$ McDonald's Omega for ordinal items.

items that were otherwise rather heterogenous in item content, reflecting a largely artefactual scale due to item keying rather than item content (Crego \& Widiger, 2014; Woods, 2006). The remaining 10 preliminary scales included at least eight items. For the nine preliminary scales that included more than eight items, we selected an optimal set of eight items using ACO (see Figure 1). Table 2 summarizes the results with regard to model fit and reliability: Model fit was acceptable for the vast majority of scales $(\mathrm{CFI}>.97$ and RMSEA $<.08$, with the exception of restricted affectivity), and McDonald's Omega of all scales was greater than $.85(M d n=.93)$. Figure 2 shows the distributions of CFA factor loadings and IRT difficulty parameters. All items had loadings greater than .50, and only three items (related to unassertiveness and attention-seeking) had loadings smaller than .70 $(M d n=.84)$. Item difficulties covered a broad spectrum of severity, ranging from -1.47 to $3.49(M d n=0.82)$. For risk aversion $(M d n=-0.24)$ and social withdrawal $(M d n=0.07)$ item difficulties were somewhat lower. Note that the selected 80 items included items from each of the 15 original constructs (see Table 1), ${ }^{1}$ but did not include any reverse-keyed items (see online Supplemental Table 2, for the full item list). Table 3 provides further information on descriptive statistics and scale intercorrelations.

There was also some evidence for the convergent and discriminant validity of the scales (see Table 4 ). With regard to PID-5 SF detachment facets, anhedonia $(r=.92)$, suspiciousness $(r=.80)$, social withdrawal $(r=.85)$, intimacy avoidance $(r=.85)$, and restricted affectivity $(r=.83)$ had their highest correlation with their corresponding PID-5 SF facet scale, and were all at least moderately associated with the remaining PID-5 SF facets $(r>.35)$. Unassertiveness $(.32<r<.53)$ and risk aversion $(.14<r<.40)$ showed lower, but still substantial associations with PID-5 SF facets. In contrast, attention-seeking, thrill-seeking, and domineering were mostly unrelated to PID-5 SF facets $(-.21<r<.17)$

With regard to the BFI-2, Extraversion was negatively correlated with the seven scales of HiTOP Detachment $(-.73<r<-.39)$ and positively correlated with the three scales of Maladaptive Extraversion $(.34<r<.46)$. With a few exceptions, the Detachment scales were all negatively correlated with Agreeableness, Conscientiousness, and Open-Mindedness, and positively correlated with Negative Emotionality. The strongest associations with big five domains emerged for unassertiveness (Extraversion, $r=$ -.73), restricted affectivity (Agreeableness, $r=-.64$ ), and anhedonia (Conscientiousness, $r=-.43$; Negative Emotionality, $r=.58$ ). Negative correlations with OpenMindedness were all very similar across the seven scales $(-.36<r<-.21)$. The results on reliability and validity of the 10 scales largely replicated in Sample 2 (see online Supplemental Tables 3 to 5).

\section{Discussion}

One important next step of the HiTOP initiative is to develop a comprehensive measure that captures individual differences in all six spectra including constructs at lower levels of the hierarchy. Here, we presented the results of the HiTOP MDSW for Detachment. We started from a list of 15 consensually defined HICs that were operationalized using 247 items and assessed in two samples via online surveys. Using a stepwise procedure involving factor analyses and ACO methods we developed ten 8-item scales that capture the two broad and largely independent factors of unipolar Detachment (anhedonia, suspiciousness, social withdrawal, intimacy avoidance, unassertiveness, risk aversion, and restricted affectivity) and Maladaptive Extraversion (attention-seeking, thrill-seeking, and domineering). Our results suggest that the 10 scales may be 

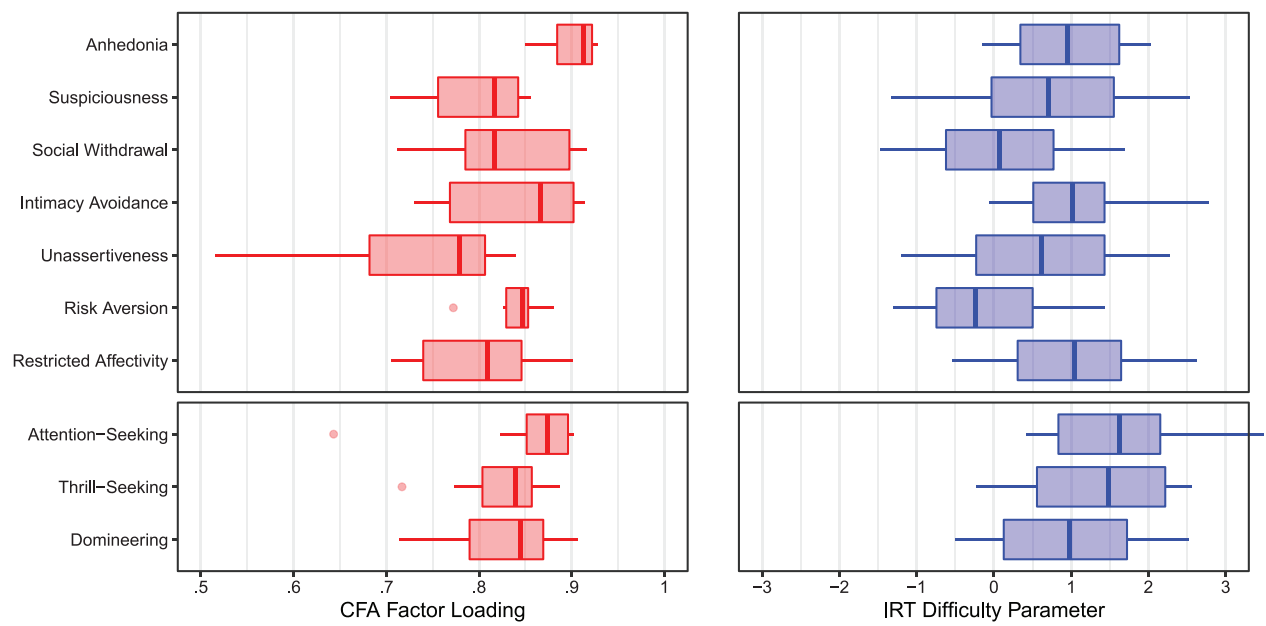

Figure 2. Boxplots of item factor loadings (based on ordinal confirmatory factor analyses) and item difficulty parameter (based on graded response models) for each of the HiTOP scales in Sample I.

Table 3. Descriptive Statistics and Intercorrelations of the HiTOP Scales in Sample I.

\begin{tabular}{|c|c|c|c|c|c|c|c|c|c|c|c|}
\hline Domain & M & $S D$ & I & 2 & 3 & 4 & 5 & 6 & 7 & 8 & 9 \\
\hline I. Anhedonia & 0.79 & 0.70 & & & & & & & & & \\
\hline 2. Suspiciousness & 0.94 & $0.7 \mathrm{I}$ & .59 & & & & & & & & \\
\hline 3. Social withdrawal & 1.42 & 0.87 & .65 & .57 & & & & & & & \\
\hline 4. Intimacy avoidance & 0.67 & 0.76 & .71 & .47 & .62 & & & & & & \\
\hline 5. Unassertiveness & 1.05 & 0.72 & .48 & .37 & .53 & .39 & & & & & \\
\hline 6. Risk aversion & 1.59 & 0.86 & .31 & .26 & .39 & .26 & .52 & & & & \\
\hline 7. Restricted affectivity & 0.77 & 0.69 & .65 & .52 & .59 & .58 & .32 & .16 & & & \\
\hline 8. Attention-seeking & 0.39 & 0.54 & -.01 & .11 & -.18 & -.05 & -.14 & -.22 & .07 & & \\
\hline 9. Thrill-seeking & 0.51 & 0.57 & .03 & .15 & -.09 & .00 & -.17 & -.58 & .16 & .56 & \\
\hline I0. Domineering & 0.78 & $0.7 \mathrm{I}$ & .00 & .15 & -.06 & -.03 & -.35 & -.23 & .15 & .58 & .43 \\
\hline
\end{tabular}

Note. $N=456$.

unidimensional, reliable, and exhibit sufficient convergent and discriminant validity to be used in Phase 2 of HiTOP data collection.

\section{Comparison With Existing Measures and Models of Detachment}

A prominent model of Detachment is featured in the DSM-5 Section III trait model (American Psychiatric Association, 2013, p. 779). Because we assessed the DSM-5 Detachment facets using the PID-5 SF, we can directly compare the preliminary HiTOP scales with the DSM-5 Section III model of Detachment. Five of the seven HiTOP scales that refer to the broad Detachment factor seem to closely correspond to five of the six DSM-5 facets, as indicated by high convergent and sufficient discriminant validity. These facets are anhedonia, suspiciousness, social withdrawal, intimacy avoidance, and restricted affectivity. The other two HiTOP scales within the broad Detachment factor (i.e., unassertiveness and risk aversion) as well as the three HiTOP scales related to Maladaptive Extraversion (i.e., attention-seeking, thrill-seeking, and domineering) are not specifically included in the DSM-5 domain of Detachment, but may be represented in other DSM-5 domains (e.g., in the domains of Negative Affectivity, Antagonism, and Disinhibition). Moreover, although we did not include the DSM-5 facet of depressivity in the list of constructs selected for HiTOP Detachment, correlations of depressivity with preliminary HiTOP scales were high, especially with anhedonia. In sum, our findings suggest that the preliminary HiTOP scales capture all relevant aspects of the DSM-5 Section III model of Detachment. 


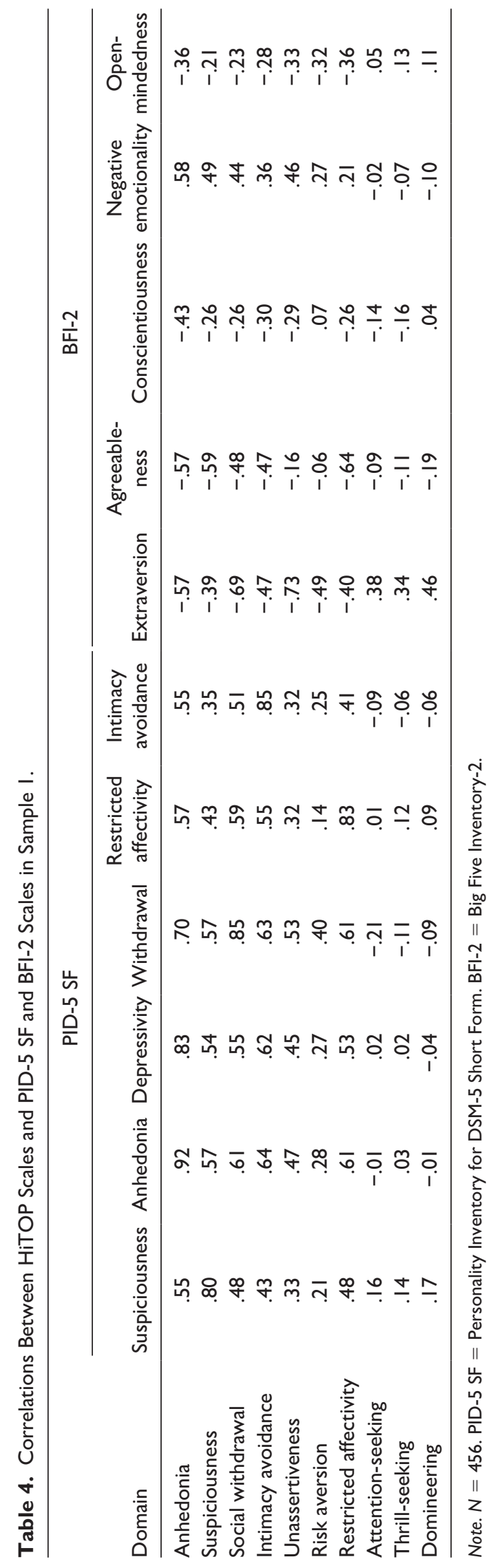


Most of the scales obtained good to acceptable convergent and discriminant relationships with the domains of the FFM. This was particularly evident for social withdrawal, intimacy avoidance, unassertiveness, risk aversion, attention-seeking, domineering, and perhaps thrill-seeking. Two clear failures were suspiciousness and restricted affectivity, which correlated more highly with low Agreeableness than with Extraversion. This is consistent with the view that suspiciousness perhaps belongs within Antagonism rather than Detachment (Watson et al., 2013; Widiger, 2011). Similarly, restricted expression of warmth and other positive feelings toward others can be perceived as a negative rejection of another person rather than simply a difficulty in expressing this affect.

\section{Unipolar Versus Bipolar Nature of Detachment}

There is considerable theoretical and empirical support for a bipolarity in maladaptive personality structure, such that there are maladaptive variants of both extraversion and introversion (Widiger \& Crego, 2019). Detachment subworkgroup members suggested including the constructs of attention-seeking (or exhibitionism), thrill-seeking, and domineering as maladaptive variants of extraversion. The Detachment and Maladaptive Extraversion scales appeared to reflect separate factors rather than occupying opposite poles of the same factor.

There are a number of possible explanations for this result (Crego et al., 2020). For the bipolar structure to emerge, one would ideally have an equal number of scales representing both poles that are truly opposite to one another. Domineering is readily understood to be opposite to unassertiveness, and thrill-seeking opposite to risk aversion, but the current study included no scales clearly opposite to anhedonia, social withdrawal, intimacy avoidance, or suspiciousness, nor clearly opposite to the Maladaptive Extraversion scale of attention-seeking. Therefore, the Maladaptive Extraversion and Detachment scales correlated substantially more positively with each other than negatively with the opposite poles (Crego et al., 2020). In addition, maladaptive scales will tend to correlate positively with other maladaptive scales (and negatively with adaptive scales) even when they involve content that is opposite to one another (Pettersson et al., 2014). This is perhaps most clearly evident when scales that are conceptually opposite to one another load in the same direction on general factors of personality and personality disorder (Pettersson et al., 2012).

It is also important to consider that many of the normal and certainly the maladaptive variants of extraversion versus introversion will occupy interstitial space (Krueger et al., 2012). This is perhaps most clearly evident for the domains of extraversion and agreeableness, FFM domains that share the same defining feature in being concerned with the manner of interpersonal relatedness (Crego et al., 2020).
Indeed, the domains of agreeableness and extraversion are arbitrary axes within the continuously distributed interpersonal circumplex structure (Pincus \& Hopwood, 2012). For example, assertiveness is a well-established trait of extraversion (Costa \& McCrae, 1992), but opposite to assertiveness would be a compliant meekness, which can be readily understood as maladaptive variants of agreeableness rather than introversion (Gore et al., 2012). In sum, it is unlikely that the bipolarity will emerge in Phase 2 as it did not appear even at this stage when the set of scales is confined to maladaptive introversion and extraversion.

\section{Locating Detachment Within HiTOP}

The location of Detachment and Maladaptive Extraversion within the structure of HiTOP is not fully clear yet. Although Detachment is currently conceptualized as a standalone spectrum, it appears that some of the preliminary scales overlap in content with scales from other spectra. For example, anhedonia has been selected as low-order construct of the Internalizing spectrum (Watson et al., in press), and risk aversion and thrill-seeking may also be located within the Internalizing spectrum when considering their associations with mania. Furthermore, because the preliminary scales of Maladaptive Extraversion were rather unrelated to Detachment, it seems likely that they will load on other spectra, such as Externalizing. We expect further discussion will be given to the inclusion of maladaptive traits opposite to Detachment, particularly as we are not aware of other HiTOP MDSW including such scales.

An even more substantial overlap exists in relation to the Thought Disorder spectrum, which has recently be subsumed together with Detachment under the rubric of a Psychosis superspectrum (Cicero et al., in press; Kotov et al., 2020). The majority of our preliminary Detachment scales (i.e., anhedonia, restricted affectivity, intimacy avoidance, suspiciousness, social withdrawal, and risk aversion) and Maladaptive Extraversion scales (i.e., attention-seeking and thrill-seeking) overlap conceptually, to some degree, with scales from the Thought Disorder spectrum. This may primarily reflect that the Thought Disorder spectrum was defined more broadly at one point to include a schizotypal spectrum rather than simply thought disorder. For example, the Thought Disorder subworkgroup included the items on seven constructs from the Detachment subworkgroup in their own scale development process (Cicero et al., in press). Therefore, it does not seem surprising that their negative psychosis symptom factor largely corresponds to the Detachment factor of our study. ${ }^{2}$ More generally, this overlap may raise questions about the core features of Detachment that justify its definition as a standalone spectrum. HiTOP Phase 2 studies that jointly assess scales from all spectra will help elucidating the discriminant validity of Detachment in more detail. 


\section{Limitations and Next Steps}

The major limitation of our study is that we did not recruit clinical samples. Thus, clinically relevant levels of Detachment or Maladaptive Extraversion were probably not very common in our samples, questioning the generalizability of our findings with regard to inpatient or outpatient populations. However, $25 \%$ of participants in Sample 1 indicated that they have received medical or psychotherapeutic treatment for mental disorders, which corresponds to findings demonstrating elevated levels of psychological distress in MTurk workers (Ophir et al., 2020; Shapiro et al., 2013). Moreover, several studies did not find major differences in the structure of psychopathology between nonclinical and clinical samples (Bach et al., 2018; O'Connor, 2002), alleviating, to some extent, concerns about generalizability. Nevertheless, it will be important for HiTOP Phase 2 studies to include samples from clinical contexts.

A further limitation involves the lack of informant or clinician reports of Detachment. Although we included two additional measures of Detachment facets and personality traits, all measures were based on self-reports, which may inflate estimates of convergent validity due to shared method variance (Podsakoff et al., 2003). Self-other agreement is known to be relatively high for Detachment compared to other maladaptive traits $(r=.50$; Oltmanns \& Oltmanns, 2021), probably because signs and symptoms of Detachment are relatively visible to observers. Nevertheless, it seems important to validate the structure of Detachment using multitrait-multimethod designs, including not only other-reports, but also ambulatory assessments of daily experiences and behaviors (e.g., Zimmermann et al., 2019). We argue that HiTOP Phase 2 studies should be complemented by such designs in selected subsamples to improve generalizability of emerging models across methods.

Our approach to developing preliminary scales for Detachment closely adhered to the guidelines of the HiTOP MDW (Simms et al., in press). That is, we prioritized the search for simple structure by using oblimin rotation in factor analyses, which is in line with the overarching aim of establishing a hierarchical model. However, alternative solutions seem possible (Condon et al., 2020), especially for the spectrum of Detachment. For example, it may be possible to use bifactor rotation and restructure the scales according to the interpersonal circumplex (Alden et al., 1990; Wilson et al., 2013). In this case, all scales would contribute to a general distress factor, and the axes of agency and communion may be defined by the poles domineering versus unassertiveness and attention-seeking versus social withdrawal, respectively. We did not test this possibility and also did not include a validation measure that could have corroborated this perspective. However, this example highlights that our results depend on various data-analytic decisions that were guided by our overarching research aims and thus we do not claim that the solution presented here represents the "only" or "true" structure of Detachment. This caution seems particularly appropriate against the background of further theoretical and methodological decisions that may limit generalizability (e.g., the definition of detachment used and the fact that only a relatively homogeneous group of four subworkgroup members could nominate constructs).

Despite these limitations, we are confident that the selected ten constructs represent the Detachment spectrum relatively comprehensively (including its theoretical maladaptive opposite) and thus form a good basis for the cross-spectrum analyses in Phase 2. Nevertheless, the selected 80 items may require some minor revisions before they can be applied in the next data collection covering all of the items from all of the spectra. For example, items should be revised to past tense and duplicate items (particularly items shared with the Thought Disorder spectrum) should be removed. Moreover, some items could be further streamlined to better match the imposed time frame of 1 year (e.g., "I have always been kind of a loner"). It might also be strategically helpful to add items for risk aversion and social withdrawal that potentially discriminate even better in future clinical samples at high severity. At the same time, due to the very high reliability of many scales, it could also be justified to further shorten the scales in Phase 2 in order to make the large number of items of all spectra manageable for participants.

\section{Authors' Note}

Christopher Conway, Leslie Morey, Thomas Widiger, and Johannes Zimmermann are members of the HiTOP Measures Development Sub-Workgroup for Detachment.

\section{Declaration of Conflicting Interests}

The author(s) declared no potential conflicts of interest with respect to the research, authorship, and/or publication of this article.

\section{Funding}

The author(s) received no financial support for the research, authorship, and/or publication of this article.

\section{ORCID iDs}

Johannes Zimmermann (iD https://orcid.org/0000-0001-6975-2356

Christopher C. Conway iD https://orcid.org/0000-0002-6848-2638

\section{Supplemental Material}

Supplemental material for this article is available online.

\section{Notes}

1. For several constructs, only very few items were retained and merged with items from other constructs to create the final scales (see Figure 1). In particular, one item from coldness was included in restricted affectivity, two items from alienation 
were included in social withdrawal, three items from autism were included in social withdrawal and restricted affectivity, three items from apathy were included in anhedonia, and two items from attention-seeking were combined with items from exhibitionism to form a new attention-seeking scale.

2. The only substantive aspect of the negative psychosis symptom factor not covered by our Detachment factor was avolition (Cicero et al., in press). This refers to physical inertia manifested by long periods without spontaneous activity, including lack of personal hygiene and self-care.

\section{References}

Alden, L. E., Wiggins, J. S., \& Pincus, A. L. (1990). Construction of circumplex scales for the Inventory of Interpersonal Problems. Journal of Personality Assessment, 55(3-4), 521536. https://doi.org/10.1080/00223891.1990.9674088

American Psychiatric Association. (2013). Diagnostic and statistical manual of mental disorders (5th ed.). Author.

Bach, B., Sellbom, M., \& Simonsen, E. (2018). Personality Inventory for DSM-5 (PID-5) in clinical versus nonclinical individuals: Generalizability of psychometric features. Assessment, 25(7), 815-825. https://doi.org/10.1177/1073191117709070

Chalmers, R. P. (2012). mirt: A multidimensional item response theory package for the R environment. Journal of Statistical Software, 48(6), 1-29. https://doi.org/10.18637/jss.v048.i06

Cicero, D. C., Jonas, K., Chmielewski, M., Martin, E. A., Docherty, A., Berzon, J., Haltigan, J. D., Reininghaus, U., Caspi, A., Grazioplene, R., \& Kotov, R. (in press). Development of the thought disorder measure for the hierarchical taxonomy of psychopathology. Assessment, Advance online publication. https://doi.org/10.1177/10731911211015355.

Clark, L. A. (1993). Manual for the schedule for nonadaptive and adaptive personality. University of Minnesota Press.

Condon, D. M., Wood, D., Mõttus, R., Booth, T., Costantini, G., Greiff, S., Johnson, W., Lukaszewski, A., Murray, A., Revelle, W., Wright, A. G. C., Ziegler, M., \& Zimmermann, J. (2020). Bottom up construction of a personality taxonomy. European Journal of Psychological Assessment, 36(6), 923-934. https://doi.org/10.1027/1015-5759/a000626

Costa, P. T., \& McCrae, R. R. (1992). Revised NEO Personality Inventory (NEO-PI-R) and NEO Five-Factor Inventory (NEOFFI) professional manual. Psychological Assessment Resources.

Crego, C., Oltmanns, J. R., \& Widiger, T. A. (2020). Obtaining and losing the bipolarity of the five-factor model through factor analysis. Personality Disorders: Theory, Research, and Treatment, 11(2), 119-130. https://doi.org/10.1037/per0000378

Crego, C., \& Widiger, T. A. (2014). Psychopathy, DSM-5, and a caution. Personality Disorders, 5(4), 335-347. https://doi. org/10.1037/per0000078

Gore, W. L., Presnall, J. R., Miller, J. D., Lynam, D. R., \& Widiger, T. A. (2012). A five-factor measure of dependent personality traits. Journal of Personality Assessment, 94(5), 488-499. https://doi.org/10.1080/00223891.2012.670681

John, O. P., Donahue, E. M., \& Kentle, R. L. (1991). The Big Five Inventory: Versions $4 a$ and 54. University of California, Berkeley, Institute of Personality and Social Research.

Kotov, R., Jonas, K. G., Carpenter, W. T., Dretsch, M. N., Eaton, N. R., Forbes, M. K., Forbush, K. T., Hobbs, K., Reininghaus,
U., Slade, T., South, S. C., Sunderland, M., Waszczuk, M. A., Widiger, T. A., Wright, A. G. C., Zald, D. H., Krueger, R. F., \& Watson, D. (2020). Validity and utility of Hierarchical Taxonomy of Psychopathology (HiTOP): I. Psychosis superspectrum. World Psychiatry, 19(2), 151-172. https://doi. org/10.1002/wps.20730

Kotov, R., Krueger, R. F., Watson, D., Achenbach, T. M., Althoff, R. R., Bagby, R. M., Brown, T. A., Carpenter, W. T., Caspi, A., Clark, L. A., Eaton, N. R., Forbes, M. K., Forbush, K. T., Goldberg, D., Hasin, D., Hyman, S. E., Ivanova, M. Y., Lynam, D. R., Markon, K., . . Zimmerman, M. (2017). The Hierarchical Taxonomy of Psychopathology (HiTOP): A dimensional alternative to traditional nosologies. Journal of Abnormal Psychology, 126(4), 454-477. https://doi. org/10.1037/abn0000258

Krueger, R. F., Derringer, J., Markon, K. E., Watson, D., \& Skodol, A. E. (2012). Initial construction of a maladaptive personality trait model and inventory for DSM-5. Psychological Medicine, 42(9), 1879-1890. https://doi.org/10.1017/S0033291711002674

Leite, W. L., Huang, I.-C., \& Marcoulides, G. A. (2008). Item selection for the development of short forms of scales using an ant colony optimization algorithm. Multivariate Behavioral Research, 43(3), 411-431. https://doi.org/10.1080/00273170802285743

Livesley, W. J., Jang, K. L., \& Vernon, P. A. (1998). Phenotypic and genetic structure of traits delineating personality disorder. Archives of General Psychiatry, 55(10), 941-948. https://doi. org/10.1001/archpsyc.55.10.941

Maples, J. L., Carter, N. T., Few, L. R., Crego, C., Gore, W. L., Samuel, D. B., Williamson, R. L., Lynam, D. R., Widiger, T. A., Markon, K. E., Krueger, R. F., \& Miller, J. D. (2015). Testing whether the DSM-5 personality disorder trait model can be measured with a reduced set of items: An item response theory investigation of the Personality Inventory for DSM5. Psychological Assessment, 27(4), 1195-1210. https://doi. org/10.1037/pas0000120

Meade, A. W., \& Craig, S. B. (2012). Identifying careless responses in survey data. Psychological Methods, 17(3), 437455. https://doi.org/10.1037/a0028085

O'Connor, B. P. (2002). The search for dimensional structure differences between normality and abnormality: A statistical review of published data on personality and psychopathology. Journal of Personality and Social Psychology, 83(4), 962-982. https://doi.org/10.1037//0022-3514.83.4.962

Olaru, G., Schroeders, U., Hartung, J., \& Wilhelm, O. (2019). Ant colony optimization and local weighted structural equation modeling: A tutorial on novel item and person sampling procedures for personality research. European Journal of Personality, 33(3), 400-419. https://doi.org/10.1002/per.2195

Oltmanns, J. R., \& Oltmanns, T. (2021). Self-other agreement on ratings of personality disorder symptoms and traits: Three meta-analyses. In T. D. Letzring \& J. S. Spain (Eds.), The handbook of accurate personality judgment: Theory and empirical findings. Oxford University Press. https://doi. org/10.1093/oxfordhb/9780190912529.013.19

Ophir, Y., Sisso, I., Asterhan, C. S. C., Tikochinski, R., \& Reichart, R. (2020). The turker blues: Hidden factors behind increased depression rates among Amazon's Mechanical Turkers. Clinical Psychological Science, 8(1), 65-83. https:// doi.org/10.1177/2167702619865973 
Pettersson, E., Mendle, J., Turkheimer, E., Horn, E. E., Ford, D. C., Simms, L. J., \& Clark, L. A. (2014). Do maladaptive behaviors exist at one or both ends of personality traits? Psychological Assessment, 26(2), 433-446. https://doi.org /10.1037/a0035587

Pettersson, E., Turkheimer, E., Horn, E. E., \& Menatti, A. R. (2012). The general factor of personality and evaluation. European Journal of Personality, 26(3), 292-302. https://doi. org/10.1002/per.839

Pincus, A. L., \& Hopwood, C. J. (2012). A contemporary interpersonal model of personality pathology and personality disorder. In T. A. Widiger (Ed.), The Oxford handbook of personality disorders (pp. 372-398). https://doi.org/10.1093/ oxfordhb/9780199735013.013.0018

Podsakoff, P. M., MacKenzie, S. B., Lee, J.-Y., \& Podsakoff, N. P. (2003). Common method biases in behavioral research: A critical review of the literature and recommended remedies. Journal of Applied Psychology, 88(5), 879-903. https://doi. org/10.1037/0021-9010.88.5.879

R Core Team. (2016). R: A language and environment for statistical computing [Computer software]. R Foundation for Statistical Computing. Vienna, Austria. https://www.R-project.org/

Revelle, W. (2018). psych: Procedures for personality and psychological research (Version 1.8.12) [Computer software]. Northwestern University, Evanston, Illinois, USA. https:// CRAN.R-project.org/package $=$ psych

Rosseel, Y. (2012). lavaan: An R package for structural equation modeling. Journal of Statistical Software, 48(2), 1-36. http:// www.jstatsoft.org/v48/i02/

Schönbrodt, F. D., \& Perugini, M. (2013). At what sample size do correlations stabilize? Journal of Research in Personality, 47(5), 609-612. https://doi.org/10.1016/j.jrp.2013.05.009

Schroeders, U., Wilhelm, O., \& Olaru, G. (2016). Meta-heuristics in short scale construction: Ant colony optimization and genetic algorithm. PLoS ONE, 11(11), Article e0167110. https://doi.org/10.1371/journal.pone.0167110

Schultze, M. (2019). stuart: Subtests using algorithmic rummaging techniques (Version 0.8.0) [Computer software]. https:// CRAN.R-project.org/package=stuart

Shapiro, D. N., Chandler, J., \& Mueller, P. A. (2013). Using Mechanical Turk to study clinical populations. Clinical Psychological Science, 1(2), 213-220. https://doi.org/10.1177 /2167702612469015

Simms, L. J., Wright, A. G. C., Cicero, D. C., Kotov, R., MullinsSweatt, S. N., Sellbom, M., Watson, D. C., Widiger, T. A., \& Zimmermann, J. (in press). Development of measures for the Hierarchical Taxonomy of Psychopathology (HiTOP): A collaborative scale development project. Assessment. Advance online publication. https://doi. org/10.1177/10731911211015309

Soto, C. J., \& John, O. P. (2017). The next Big Five Inventory (BFI-2): Developing and assessing a hierarchical model with 15 facets to enhance bandwidth, fidelity, and predictive power. Journal of Personality and Social Psychology, 113(1), 117-143. https://doi.org/10.1037/pspp0000096

Watson, D., Forbes, M. K., Levin-Aspenson, H. F., Ruggero, C. J., Kotelnikova, Y., Khoo, S., Bagby, R. M., Sunderland, M., Patalay, P., \& Kotov, R. (in press). The development of preliminary HiTOP internalizing spectrum scales. Assessment. Advance online publication. https://doi.org /10.1177/10731911211003976

Watson, D., Stasik, S. M., Ro, E., \& Clark, L. A. (2013). Integrating normal and pathological personality: Relating the DSM-5 trait-dimensional model to general traits of personality. Assessment, 20(3), 312-326. https://doi.org $/ 10.1177 / 1073191113485810$

Widiger, T. A. (2011). The DSM-5 dimensional model of personality disorder: Rationale and empirical support. Journal of Personality Disorders, 25(2), 222-234. https://doi.org /10.1521/pedi.2011.25.2.222

Widiger, T. A., \& Crego, C. (2019). The bipolarity of normal and abnormal personality structure: Implications for assessment. Psychological Assessment, 31(4), 420-431. https://doi. org/10.1037/pas0000546

Widiger, T. A., Lynam, D. R., Miller, J. D., \& Oltmanns, T. F. (2012). Measures to assess maladaptive variants of the five-factor model. Journal of Personality Assessment, 94(5), 450-455. https://doi.org/10.1080/00223891.2012.6 77887

Wilson, S., Revelle, W., Stroud, C. B., \& Durbin, C. E. (2013). A confirmatory bifactor analysis of the Inventory of Interpersonal Problems - Circumplex and associations of interpersonal traits across multiple relationship contexts and measures. Psychological Assessment, 25(2), 353-365. https://doi.org/10.1037/a0030989

Woods, C. M. (2006). Careless responding to reverse-worded items: Implications for confirmatory factor analysis. Journal of Psychopathology and Behavioral Assessment, 28(3), 186-191. https://doi.org/10.1007/s10862-005-9004-7

World Health Organization. (2018). ICD-11 for mortality and morbidity statistics (ICD-11 MMS). https://icd.who.int

Wright, A. G. C., \& Simms, L. J. (2014). On the structure of personality disorder traits: Conjoint analyses of the CAT-PD, PID-5, and NEO-PI-3 trait models. Personality Disorders: Theory, Research, and Treatment, 5(1), 43-54. https://doi. org/10.1037/per0000037

Zimmermann, J., Woods, W. C., Ritter, S., Happel, M., Masuhr, O., Jaeger, U., Spitzer, C., \& Wright, A. G. C. (2019). Integrating structure and dynamics in personality assessment: First steps toward the development and validation of a personality dynamics diary. Psychological Assessment, 31(4), 516-531. https://doi.org/10.1037/pas 0000625 